\title{
ON SINGULAR ELLIPTIC EQUATIONS WITH MEASURE SOURCES
}

\author{
FRANCESCANTONIO OLIVA AND FRANCESCO PETITTA
}

\begin{abstract}
We prove existence of solutions for a class of singular elliptic problems with a general measure as source term whose model is$$
\begin{cases}-\Delta u=\frac{f(x)}{u^{\gamma}}+\mu & \text { in } \Omega, \\ u=0 & \text { on } \partial \Omega, \\ u>0 & \text { on } \Omega,\end{cases}
$$

where $\Omega$ is an open bounded subset of $\mathbb{R}^{N}$. Here $\gamma>0, f$ is a nonnegative function on $\Omega$, and $\mu$ is a nonnegative bounded Radon measure on $\Omega$.
\end{abstract}

\section{INTRODUCTION}

In this paper we prove existence of a nonnegative weak solution to the following elliptic problem

$$
\begin{cases}-\operatorname{div}(A(x) \nabla u)=\frac{f(x)}{u^{\gamma}}+\mu & \text { in } \Omega, \\ u=0 & \text { on } \partial \Omega,\end{cases}
$$

where $\Omega$ is an open bounded subset of $\mathbb{R}^{N}, N \geq 2, \gamma>0, f$ is a nonnegative function which has some Lebesgue regularity and $\mu$ is a nonnegative bounded Radon measure. Here $A: \Omega \rightarrow \mathbb{R}^{N^{2}}$ is a bounded elliptic matrix, and we ask both $f$ and $\mu$ to be not identically zero. We stress that the problem is singular as one ask to the solution to be zero on the boundary.

If $\mu \equiv 0, A(x) \equiv I$, and $f$ is smooth, then problem (1.1) has been extensively studied in the past (see for instance [17, 23] and references therein). Recently, the existence of a distributional solution for problem (1.1) has been considered in 8 again in the homogeneous case $\mu \equiv 0$ (see also 6 , 3 for further improvements).

The nonhomogeneous case (i.e. $\mu \neq 0$ ) has also been considered: motivated by the study of $G$-convergence in periodic composite media, in [20, 21] the authors prove existence of bounded solutions to problems (1.1) if both $f$ and $\mu$ belong to $L^{q}(\Omega)$, with $q>\frac{N}{2}$, while in [15] symmetry properties for solutions of a related semilinear problem are considered (see also [14, 13, 16, for further related results).

Let us also observe that problem (1.1) is also related, through the standard Hopf-Lax type transformation $u=v^{1-\eta}$ (with $\eta=\frac{\gamma}{\gamma+1}$ ) to the singular quadratic problem (for simplicity, consider $A(x)=I$ )

$$
\begin{cases}-\Delta v+\eta \frac{|\nabla v|^{2}}{v}=\tilde{g}(x) v^{\eta}+\tilde{f}(x) & \text { in } \Omega, \\ v=0 & \text { on } \partial \Omega\end{cases}
$$

with $\tilde{g}=\frac{\mu}{1-\eta}$ and $\tilde{f}=\frac{f}{1-\eta}$. If $\tilde{g} \equiv 0$ and $f$ is an $L^{1}$ function then problem (1.2) has been recently considered in the literature (see for instance 4, 1, 22, and references therein). Finally, the case of $\tilde{g}$ being a constant and $\tilde{f} \in L^{\frac{2 N}{N+2}}(\Omega)$ has been studied in [2] through variational methods. In particular, Theorem 2.14 below can be viewed as a generalization of the existence result in [2, Theorem 1.2].

Our aim is to give a complete account on the solvability of boundary value problems as (1.1) under minimal assumptions on the data. The plan of the paper is the following: Section 2 is devoted to the proof of existence of weak solutions for problem (1.1) in the general case through an approximation argument. In Section 2.4 we further investigate a special case of (1.1) (i.e. $A(x)=I$ and $\gamma<1$ ) on smooth domains; in this case we prove

2000 Mathematics Subject Classification. 35J60, 35J61, 35J75, 35R06.

Key words and phrases. Nonlinear elliptic equations, Singular elliptic equations, Measure data. 
existence and uniqueness of suitable solutions to (1.1). Finally, in Section 3 we show how to generalize some of the results we obtained to the case of a nonlinear principal part of Leray-Lions type with growth $p-1$ as the $p$-laplacian.

Notations. If no otherwise specified, we will denote by $C$ several constants whose value may change from line to line and, sometimes, on the same line. These values will only depend on the data (for instance $C$ can depend on $\Omega, \gamma, N, \alpha$ and $\beta$ ) but they will never depend on the indexes of the sequences we will often introduce. For the sake of simplicity we will often use the simplified notation

$$
\int_{\Omega} f:=\int_{\Omega} f(x) d x
$$

when referring to integrals when no ambiguity on the variable of integration is possible.

For fixed $k>0$ we will made use of the truncation functions $T_{k}$ and $G_{k}$ defined as

$$
T_{k}(s)=\max (-k, \min (s, k)),
$$

and

$$
G_{k}(s)=(|s|-k)^{+} \operatorname{sign}(s) .
$$

We will also make use of the Marcinkievicz space $M^{q}(\Omega)$ (or weak $L^{q}(\Omega)$ ) which is defined, for every $0<q<$ $\infty$, as the space of all measurable functions $f$ such that there exists $c>0$ with

$$
m(\{x:|f(x)|>t\}) \leq \frac{c}{t^{q}}
$$

Of course, as $\Omega$ is bounded, then it suffices for the previous inequality to hold for $t \geq t_{0}$, for some fixed positive $t_{0}$. We only recall that the following continuous embeddings hold

$$
L^{q}(\Omega) \hookrightarrow M^{q}(\Omega) \hookrightarrow L^{q-\epsilon}(\Omega),
$$

for every $1<q<\infty$ and $0<\epsilon \leq q-1$.

\section{Main assumptions And existence of a solution}

Let us consider the following boundary value problem

$$
\begin{cases}-\operatorname{div}(A(x) \nabla u)=\frac{f(x)}{u^{\gamma}}+\mu & \text { in } \Omega, \\ u=0 & \text { on } \partial \Omega,\end{cases}
$$

where $\Omega$ is any open bounded subset of $\mathbb{R}^{N}, N \geq 2, \gamma>0, f$ is a nonnegative function in $L^{1}(\Omega)$, $\mu$ is a nonnegative bounded Radon measure, and $A: \Omega \rightarrow \mathbb{R}^{N^{2}}$ is a bounded elliptic matrix, which satisfies

$$
\alpha|\xi|^{2} \leq A(x) \xi \cdot \xi, \quad|A(x)| \leq \beta,
$$

for every $\xi$ in $\mathbb{R}^{N}$, for almost every $x$ in $\Omega$, for some $0<\alpha \leq \beta$. As we said we ask to both $f$ and $\mu$ to be not identically zero otherwise we fall back in the previous considered cases of, respectively, 28] and [8].

In this kind of generality, uniqueness of weak solutions is not expected in general even in smoother cases (we refer to [1] for further comments on this fact). In Section 2.4 below we will prove a uniqueness result in a very special model case (i.e. $A(x)=I$ and $\gamma<1$ ).

Our aim is to prove the existence of suitable weak solutions to (2.4). Here is the notion of solution we will consider.

Definition 2.1. If $\gamma \leq 1$, then a weak solution to problem (2.4) is a function $u \in W_{0}^{1,1}(\Omega)$ such that

$$
\forall \omega \subset \subset \exists \exists c_{\omega}: u \geq c_{\omega}>0,
$$

and such that

$$
\int_{\Omega} A(x) \nabla u \cdot \nabla \varphi=\int_{\Omega} \frac{f \varphi}{u^{\gamma}}+\int_{\Omega} \varphi d \mu, \quad \forall \varphi \in C_{c}^{1}(\Omega) .
$$

If $\gamma>1$, then a weak solution to problem (2.4) is a function $u \in W_{l o c}^{1,1}(\Omega)$ satisfying (2.5), (2.6), and such that $T_{k}^{\frac{\gamma+1}{2}}(u) \in H_{0}^{1}(\Omega)$ for every fixed $k>0$. 
Remark 2.2. First of all observe that, due to (2.5), all terms in (2.6) are well defined. Let us spend some words on how the boundary data is intended in the strongly singular case (i.e. $\gamma>1$ ). In fact, in this case the Dirichlet datum is not expected to be achieved in the classical sense of traces. Even in the case $\mu=0$, this was already noticed in [23; in this paper the authors proved that, if $f$ is a bounded smooth function then no solutions in $H_{0}^{1}(\Omega)$ can be found if $\gamma \geq 3$. The threshold 3 is essentially due to the fact that the datum $f$ is assumed to be bounded. A sharper result was recently proven, through variational methods, in 29, Theorem 1] for a nonnegative $f \in L^{1}(\Omega)$ and $A(x)=I$. This result reads as: an $H_{0}^{1}(\Omega)$ solution does exist for problem

$$
\begin{cases}-\Delta u=\frac{f(x)}{u^{\gamma}} & \text { in } \Omega \\ u=0 & \text { on } \partial \Omega\end{cases}
$$

if and only if there exist a function $u_{0} \in H_{0}^{1}(\Omega)$ such that

$$
\int_{\Omega} f u_{0}^{1-\gamma} d x<\infty
$$

A straightforward computation shows that, at least in the radial case, for any $\gamma>1$ condition (2.7) is not satisfied for suitable $f \in L^{1}(\Omega)$. For that reason we need to impose the weaker condition on the truncations of $u$ (i.e. $T(u)^{\frac{\gamma+1}{2}} \in H_{0}^{1}(\Omega)$ ) in order to give a (weak) sense to the boundary datum. This kind of weak boundary condition was already used in the literature (see for instance [8]). Here we need to use truncations of $u$ as the presence of the measure data $\mu$ does not allow to conclude that $u^{\frac{\gamma+1}{2}}$ itself belongs to $H_{0}^{1}(\Omega)$. Anyway let us observe that, if $\Omega$ is smooth enough and $v^{\frac{\gamma+1}{2}} \in H_{0}^{1}(\Omega)$ ( $v$ being a nonnegative function), then

$$
\lim _{\varepsilon \rightarrow 0^{+}} \frac{1}{\varepsilon} \int_{\{x: \operatorname{dist}(x, \partial \Omega)<\varepsilon\}} v^{\frac{\gamma+1}{2}}(x) d x=0
$$

(see [26]). As $\gamma>1$, using Hölder's inequality one can easily get that

$$
\lim _{\varepsilon \rightarrow 0^{+}} \frac{1}{\varepsilon} \int_{\{x: \operatorname{dist}(x, \partial \Omega)<\varepsilon\}} v(x) d x=0,
$$

which is a clearer way to understand the boundary condition we use here.

We will prove existence of solutions for problem (2.4) by approximation. In order to do that we need some preliminary results on the approximating sequences of solutions that can be proven no matter of the value of $\gamma$.

Let us consider the following problem

$$
\begin{cases}-\operatorname{div}\left(A(x) \nabla u_{n}\right)=\frac{f_{n}}{\left(u_{n}+\frac{1}{n}\right)^{\gamma}}+\mu_{n} & \text { in } \Omega \\ u_{n}=0 & \text { on } \partial \Omega\end{cases}
$$

where $f_{n}$ is the truncation at level $n$ of $f$ and $\mu_{n}$ is a sequence of smooth nonnegative functions, bounded in $L^{1}(\Omega)$, converging weakly to $\mu$ in the sense of the measures.

We want to prove the existence of a weak solution of problem (2.8) for every fixed $n \in \mathbb{N}$.

Lemma 2.3. Problem (2.8) admits a nonnegative weak solution $u_{n} \in H_{0}^{1}(\Omega) \cap L^{\infty}(\Omega)$.

Proof. The proof is based on standard Schauder's fixed point argument. Let $n \in \mathbb{N}$ be fixed, let us define

$$
G: L^{2}(\Omega) \rightarrow L^{2}(\Omega)
$$

as the map that, for any $v \in L^{2}(\Omega)$ gives the weak solution $w$ to the following problem

$$
\begin{cases}-\operatorname{div}(A(x) \nabla w)=\frac{f_{n}}{\left(|v|+\frac{1}{n}\right)^{\gamma}}+\mu_{n} & \text { in } \Omega, \\ w=0 & \text { on } \partial \Omega .\end{cases}
$$

It follows from classical theory (e.g. by Lax-Milgram lemma) that $w \in H_{0}^{1}(\Omega)$ for every fixed $v \in L^{2}(\Omega)$. This implies that we can choose $w$ as test function in the weak formulation. 
Thus

$$
\alpha \int_{\Omega}|\nabla w|^{2} \leq \int_{\Omega} A(x) \nabla w \cdot \nabla w=\int_{\Omega} \frac{f_{n} w}{\left(|v|+\frac{1}{n}\right)^{\gamma}}+\int_{\Omega} w \mu_{n} \leq\left(n^{\gamma+1}+C(n)\right) \int_{\Omega}|w| \cdot
$$

Therefore, using the Poincaré inequality on the left hand side and the Hölder inequality on the right side

This means that

$$
\int_{\Omega}|w|^{2} \leq C^{\prime}\left(n^{\gamma+1}+C(n)\right)\left(\int_{\Omega}|w|^{2}\right)^{\frac{1}{2}} \text {. }
$$

$$
\|w\|_{L^{2}(\Omega)} \leq C^{\prime}\left(n^{\gamma+1}+C(n)\right)
$$

where $C^{\prime}, C(n)$ are independent from $v$. Thus, we have that the ball of radius $C^{\prime}\left(n^{\gamma+1}+C(n)\right)$ is invariant for $G$. Now we prove that the map $G$ is continuous in $L^{2}(\Omega)$. Let us choose a sequence $v_{k}$ that converges to $v$ in $L^{2}(\Omega)$; then, by the dominated convergence theorem

$$
\left(\frac{f_{n}}{\left(\left|v_{k}\right|+\frac{1}{n}\right)^{\gamma}}+\mu_{n}\right)_{k \in \mathbb{N}} \text { converges to }\left(\frac{f_{n}}{\left(|v|+\frac{1}{n}\right)^{\gamma}}+\mu_{n}\right) \text { in } L^{2}(\Omega) .
$$

Thus, by the uniqueness of the weak solution for the linear problem, we can say that $w_{k}:=G\left(v_{k}\right)$ converges to $w:=G(v)$ in $L^{2}(\Omega)$. Therefore, we proved that $G$ is continuous.

What finally need to check that the set $G\left(L^{2}(\Omega)\right)$ is relatively compact in $L^{2}(\Omega)$. We proved before that

$$
\int_{\Omega}|\nabla G(v)|^{2} \leq C(n, \gamma)
$$

for any $v \in L^{2}(\Omega)$, so that, $G(v)$ is relatively compact in $L^{2}(\Omega)$ by Rellich-Kondrachov theorem.

Now we can finally apply Schauder fixed point theorem to obtain that $G$ has a fixed point $u_{n} \in L^{2}(\Omega)$ that is a solution to (2.8) in $H_{0}^{1}(\Omega)$. Moreover, $u_{n}$ belongs to $L^{\infty}(\Omega)$ (see [28]). Here $\left(\frac{f_{n}}{\left(\left|u_{n}\right|+\frac{1}{n}\right)^{\gamma}}+\mu_{n}\right) \geq 0$ then the maximum principle implies that $u_{n} \geq 0$ and this concludes the proof.

The next step consists in the proof that $u_{n}$ is uniformly bounded from below on the compact subsets of $\Omega$.

Lemma 2.4. The sequence $u_{n}$ is such that for every $\omega \subset \subset \Omega$ there exists $c_{\omega}$ (not depending on $n$ ) such that

$$
u_{n}(x) \geq c_{\omega}>0 \text {, for a.e. } x \text { in } \omega \text {, and for every } n \text { in } \mathbb{N} \text {. }
$$

Proof. Let consider the following problem

$$
\begin{cases}-\operatorname{div}\left(A(x) \nabla v_{n}\right)=\frac{f_{n}}{\left(v_{n}+\frac{1}{n}\right)^{\gamma}} & \text { in } \Omega \\ v_{n}=0 & \text { on } \partial \Omega .\end{cases}
$$

It was proved in [8] the existence of a weak solution $v_{n}$ of (2.9) such that

$$
\forall \omega \subset \subset \Omega \exists c_{\omega}: v_{n} \geq c_{\omega}>0,
$$

for almost every $x$ in $\omega$ and where $c_{\omega}$ is independent of $n$. Thus, taking $\left(u_{n}-v_{n}\right)^{-}$as test function in the difference between the formulations of, respectively, (2.8) and (2.9), we obtain, using ellipticity

$$
\begin{aligned}
& -\alpha \int_{\Omega}\left|\nabla\left(u_{n}-v_{n}\right)^{-}\right|^{2} \geq \int_{\Omega} A(x) \nabla\left(u_{n}-v_{n}\right) \cdot \nabla\left(u_{n}-v_{n}\right)^{-} \\
& =\int_{\Omega}\left(\frac{f_{n}(x)}{\left(u_{n}+\frac{1}{n}\right)^{\gamma}}-\frac{f_{n}(x)}{\left(v_{n}+\frac{1}{n}\right)^{\gamma}}\right)\left(u_{n}-v_{n}\right)^{-}+\int_{\Omega} \mu_{n}\left(u_{n}-v_{n}\right)^{-} \geq 0,
\end{aligned}
$$

that implies $u_{n} \geq v_{n}$ for a.e. $x$ in $\omega$, and so

$$
\forall \omega \subset \subset \exists \exists c_{\omega}: u_{n} \geq c_{\omega}>0, \text { for a.e. } x \text { in } \omega .
$$

We can now prove existence of solutions for problem (2.4). In order to do that we distinguish between two cases. 
2.1. The case $\gamma \leq 1$. As one could expect from classical theory of measure data problem we will have that $u_{n}$ is bounded in $W_{0}^{1, q}(\Omega)$ for every $q<\frac{N}{N-1}$.

Lemma 2.5. Let $u_{n}$ be the solution of (2.8) with $\gamma \leq 1$. Then $u_{n}$ is bounded in $W_{0}^{1, q}(\Omega)$ for every $q<\frac{N}{N-1}$.

Proof. We follow the classical approach of [5]. We will prove that $\nabla u_{n}$ is bounded in $M^{\frac{N}{N-1}}(\Omega)$. Without loss of generality we can suppose $N>2$. The case $N=2$ is easier and can be treated in a standard way with many simplifications.

We have

$$
\begin{aligned}
& \left\{\left|\nabla u_{n}\right| \geq t\right\}=\left\{\left|\nabla u_{n}\right| \geq t, u_{n}<k\right\} \cup\left\{\left|\nabla u_{n}\right| \geq t, u_{n} \geq k\right\} \\
& \subset\left\{\left|\nabla u_{n}\right| \geq t, u_{n}<k\right\} \cup\left\{u_{n} \geq k\right\}
\end{aligned}
$$

thus,

$$
m\left(\left\{\left|\nabla u_{n}\right| \geq t\right\}\right) \leq m\left(\left\{\left|\nabla u_{n}\right| \geq t, u_{n}<k\right\}\right)+m\left(\left\{u_{n} \geq k\right\}\right)
$$

We take $\varphi=\left(T_{k}\left(u_{n}\right)+\epsilon\right)^{\gamma}-\epsilon^{\gamma}$ as test function in the weak formulation of (2.8) where $\epsilon>0$. We stress that the $\epsilon$ perturbation of $T_{k}\left(u_{n}\right)^{\gamma}$ is only needed in the case $\gamma<1$ in order to be allowed to use it as test function. We obtain,

$$
\alpha \int_{\Omega}\left|\nabla T_{k}\left(u_{n}\right)\right|^{2}\left(T_{k}\left(u_{n}\right)+\epsilon\right)^{\gamma-1} \leq \int_{\Omega} \frac{f_{n}\left[\left(T_{k}\left(u_{n}\right)+\epsilon\right)^{\gamma}-\epsilon^{\gamma}\right]}{\left(u_{n}+\frac{1}{n}\right)^{\gamma}}+\int_{\Omega}\left[\left(T_{k}\left(u_{n}\right)+\epsilon\right)^{\gamma}-\epsilon^{\gamma}\right] \mu_{n} .
$$

For fixed $n$, if $\epsilon$ is taken smaller than $\frac{1}{n}$, then we can say that $\frac{\left[\left(T_{k}\left(u_{n}\right)+\epsilon\right)^{\gamma}-\epsilon^{\gamma}\right]}{\left(u_{n}+\frac{1}{n}\right)^{\gamma}} \leq 1$. Thus, for the right hand side of (2.10) we have both

$$
\int_{\Omega} \frac{f_{n}\left[\left(T_{k}\left(u_{n}\right)+\epsilon\right)^{\gamma}-\epsilon^{\gamma}\right]}{\left(u_{n}+\frac{1}{n}\right)^{\gamma}} \leq \int_{\Omega} f_{n} \leq\|f\|_{L^{1}(\Omega)}
$$

and

$$
\int_{\Omega}\left[\left(T_{k}\left(u_{n}\right)+\epsilon\right)^{\gamma}-\epsilon^{\gamma}\right] \mu_{n} \leq(k+\epsilon)^{\gamma}\left\|\mu_{n}\right\|_{L^{1}(\Omega)} .
$$

Combining the previous results we obtain

$$
\int_{\Omega}\left|\nabla T_{k}\left(u_{n}\right)\right|^{2}\left(T_{k}\left(u_{n}\right)+\epsilon\right)^{\gamma-1} \leq C\left[1+(k+\epsilon)^{\gamma}\right] .
$$

Therefore,

$$
\begin{aligned}
& \int_{\Omega}\left|\nabla T_{k}\left(u_{n}\right)\right|^{2}=\int_{\Omega} \frac{\left|\nabla T_{k}\left(u_{n}\right)\right|^{2}}{\left(T_{k}\left(u_{n}\right)+\epsilon\right)^{\gamma-1}}\left(T_{k}\left(u_{n}\right)+\epsilon\right)^{\gamma-1} \\
& \leq\left.\left.(k+\epsilon)^{1-\gamma}|| \nabla T_{k}\left(u_{n}\right)\right|^{2}\left(T_{k}\left(u_{n}\right)+\epsilon\right)^{\gamma-1}\right|_{L^{1}(\Omega)} \leq C(k+\epsilon)^{1-\gamma}\left[1+(k+\epsilon)^{\gamma}\right],
\end{aligned}
$$

and passing to the limit in $\epsilon$

$$
\int_{\Omega}\left|\nabla T_{k}\left(u_{n}\right)\right|^{2} \leq C\left(k^{1-\gamma}+k\right) .
$$

Let us observe that the constant $C$ does not depend on the index $n$ of the sequence. Thus, it follows from the Sobolev inequality that

$$
\frac{1}{\mathcal{S}^{2}}\left(\int_{\Omega}\left|T_{k}\left(u_{n}\right)\right|^{2^{*}}\right)^{\frac{2}{2^{*}}} \leq \int_{\Omega}\left|\nabla T_{k}\left(u_{n}\right)\right|^{2} \leq C\left(k^{1-\gamma}+k\right) .
$$

If we restrict the integral on the left hand side on $\left\{u_{n} \geq k\right\}$ (on which $T_{k}\left(u_{n}\right)=k$ ) we then obtain

$$
k^{2} m\left(\left\{u_{n} \geq k\right\}\right)^{\frac{2}{2^{*}}} \leq C\left(k^{1-\gamma}+k\right),
$$


so that

$$
m\left(\left\{u_{n} \geq k\right\}\right) \leq C\left(\frac{\left(k^{1-\gamma}+k\right)}{k^{2}}\right)^{\frac{N}{N-2}} \leq \frac{C}{k^{\frac{N}{N-2}}} \quad \forall k \geq 1
$$

that is $u_{n}$ is bounded in $M^{\frac{N}{N-2}}(\Omega)$.

Now we estimate $m\left(\left\{\left|\nabla u_{n}\right| \geq t, u_{n}<k\right\}\right)$. From (2.11) we have

$$
m\left(\left\{\left|\nabla u_{n}\right| \geq t, u_{n}<k\right\}\right) \leq \frac{1}{t^{2}} \int_{\Omega}\left|\nabla T_{k}\left(u_{n}\right)\right|^{2} \leq \frac{C\left(k^{1-\gamma}+k\right)}{t^{2}} \leq \frac{C k}{t^{2}} \quad \forall k \geq 1 .
$$

Combining previous inequalities we obtain

$$
m\left(\left\{\left|\nabla u_{n}\right| \geq t\right\}\right) \leq m\left(\left\{\left|\nabla u_{n}\right| \geq t, u_{n}<k\right\}\right)+m\left(\left\{u_{n} \geq k\right\}\right) \leq \frac{C k}{t^{2}}+\frac{C}{k^{\frac{N}{N-2}}} \quad \forall k \geq 1 .
$$

We then choose $k=t^{\frac{N-2}{N-1}}$

$$
m\left(\left\{\left|\nabla u_{n}\right| \geq t\right\}\right) \leq \frac{C t^{\frac{N-2}{N-1}}}{t^{2}}+\frac{C}{t^{\frac{N}{N-1}}} \quad \forall t \geq 1 .
$$

Thus we get for a constant $C$ not depending on $n$

$$
m\left(\left\{\left|\nabla u_{n}\right| \geq t\right\}\right) \leq \frac{C}{t^{\frac{N}{N-1}}} \quad \forall t \geq 1 .
$$

We have just proved that $\nabla u_{n}$ is bounded in $M^{\frac{N}{N-1}}(\Omega)$. This implies by property (1.3) that $u_{n}$ is bounded in $W_{0}^{1, q}(\Omega)$ for every $q<\frac{N}{N-1}$.

We now can prove our existence result for $\gamma \leq 1$.

Theorem 2.6. Let $\gamma \leq 1$. Then there exists a weak solution $u$ of 2.4) in $W_{0}^{1, q}(\Omega)$ for every $q<\frac{N}{N-1}$.

Proof. It follows from Lemma 2.5 that there exists $u$ such that (up to not relabeled subsequences) the sequence $u_{n}$ converges weakly to $u$ in $W_{0}^{1, q}(\Omega)$ for every in $q<\frac{N}{N-1}$. This implies that for $\varphi$ in $C_{c}^{1}(\Omega)$

$$
\lim _{n \rightarrow+\infty} \int_{\Omega} A(x) \nabla u_{n} \cdot \nabla \varphi=\int_{\Omega} A(x) \nabla u \cdot \nabla \varphi .
$$

Moreover, by compact embeddings, we can also assume that $u_{n}$ converges to $u$ both strongly in $L^{1}(\Omega)$ and a.e. Thus, taking $\varphi$ in $C_{c}^{1}(\Omega)$, we have that

$$
0 \leq\left|\frac{f_{n} \varphi}{\left(u_{n}+\frac{1}{n}\right)^{\gamma}}\right| \leq \frac{\|\varphi\|_{L^{\infty}(\Omega)}}{c_{\omega}} f
$$

where $\omega$ is the set $\{\varphi \neq 0\}$. This is enough to apply the dominated convergence theorem so that

$$
\lim _{n \rightarrow+\infty} \int_{\Omega} \frac{f_{n} \varphi}{\left(u_{n}+\frac{1}{n}\right)^{\gamma}}=\int_{\Omega} \frac{f \varphi}{u^{\gamma}} .
$$

This concludes the proof of the result as it is straightforward to pass to the limit in the last term involving $\mu_{n}$.

2.2. The strongly singular case: $\gamma>1$. In this case, as we already mentioned, we can only hold some local estimates on $u_{n}$ in the Sobolev space. In order to give sense to the function $u$ on the boundary of $\Omega$, at least a weak sense, we shall provide global estimates on $T_{k}^{\frac{\gamma+1}{2}}\left(u_{n}\right)$ in $H_{0}^{1}(\Omega)$.

As in the previous case we consider the solutions of the approximating solutions of (2.8). Here is our global estimate on the power of the truncation of $u_{n}$.

Lemma 2.7. Let $u_{n}$ be the solution of (2.8) with $\gamma>1$. Then $T_{k}^{\frac{\gamma+1}{2}}\left(u_{n}\right)$ is bounded in $H_{0}^{1}(\Omega)$ for every fixed $k>0$. 
Proof. We take $\varphi=T_{k}^{\gamma}\left(u_{n}\right)$ as a test function in (2.8) and we have

$$
\gamma \int_{\Omega} A(x) \nabla u_{n} \cdot \nabla T_{k}\left(u_{n}\right) T_{k}^{\gamma-1}\left(u_{n}\right)=\int_{\Omega} \frac{f_{n} T_{k}^{\gamma}\left(u_{n}\right)}{\left(u_{n}+\frac{1}{n}\right)^{\gamma}}+\int_{\Omega} T_{k}^{\gamma}\left(u_{n}\right) \mu_{n} .
$$

Using ellipticity we can estimate the term on the left hand side of (2.12) as

$$
\gamma \int_{\Omega} A(x) \nabla u_{n} \cdot \nabla T_{k}\left(u_{n}\right) T_{k}^{\gamma-1}\left(u_{n}\right) \geq \alpha \gamma \int_{\Omega}\left|\nabla T_{k}^{\frac{\gamma+1}{2}}\left(u_{n}\right)\right|^{2} .
$$

Recalling that $\frac{T_{k}^{\gamma}\left(u_{n}\right)}{\left(u_{n}+\frac{1}{n}\right)^{\gamma}} \leq \frac{u_{n}^{\gamma}}{\left(u_{n}+\frac{1}{n}\right)^{\gamma}} \leq 1$, then for the term on the right hand side of (2.12) we can write

$$
\int_{\Omega} \frac{f_{n} T_{k}^{\gamma}\left(u_{n}\right)}{\left(u_{n}+\frac{1}{n}\right)^{\gamma}}+\int_{\Omega} T_{k}^{\gamma}\left(u_{n}\right) \mu_{n} \leq 1+k^{\gamma} \leq C
$$

so that combining the previous inequalities we get

$$
\int_{\Omega}\left|\nabla T_{k}^{\frac{\gamma+1}{2}}\left(u_{n}\right)\right|^{2} \leq C
$$

that is what we had to prove.

Now, in order to pass to the limit in the weak formulation, we only need to prove some local estimates on $u_{n}$. We prove the following

Lemma 2.8. Let $u_{n}$ be the solution of (2.8) with $\gamma>1$. Then $u_{n}$ is bounded in $W_{l o c}^{1, q}(\Omega)$ for every $q<\frac{N}{N-1}$. Proof. We divide the proof in two steps.

Step 1. $G_{1}\left(u_{n}\right)$ is bounded in $W_{0}^{1, q}(\Omega)$ for every $q<\frac{N}{N-1}$.

We need to prove that

$$
\int_{\left\{u_{n}>1\right\}}\left|\nabla u_{n}\right|^{q} \leq C,
$$

where $q<\frac{N}{N-1}$. Analogously to the case $\gamma<1$, we have to prove that $\nabla G_{1}\left(u_{n}\right)$ is bounded in $M^{\frac{N}{N-1}}(\Omega)$. We have

$$
m\left(\left\{\left|\nabla u_{n}\right|>t, u_{n}>1\right\}\right) \leq m\left(\left\{\left|\nabla u_{n}\right|>t, 1<u_{n} \leq k\right\}\right)+m\left(\left\{u_{n}>k\right\}\right) .
$$

In order to estimate (2.14) we take $\varphi=T_{k}\left(G_{1}\left(u_{n}\right)\right)(k>1)$ as a test function in (2.8).

Recalling that $\nabla T_{k}\left(G_{1}\left(u_{n}\right)\right)=\nabla u_{n}$ only when $1<u_{n} \leq k$ (otherwise is zero), and that $T_{k}\left(G_{1}\left(u_{n}\right)\right)=0$ on $\left\{u_{n} \leq 1\right\}$, we have

$$
\alpha \int_{\Omega}\left|\nabla T_{k}\left(G_{1}\left(u_{n}\right)\right)\right|^{2} \leq \int_{\Omega} \frac{f_{n} T_{k}\left(G_{1}\left(u_{n}\right)\right)}{\left(1+\frac{1}{n}\right)^{\gamma}}+\int_{\Omega} T_{k}\left(G_{1}\left(u_{n}\right)\right) \mu_{n} \leq C k
$$

and

so that,

$$
\begin{aligned}
& \int_{\Omega}\left|\nabla T_{k}\left(G_{1}\left(u_{n}\right)\right)\right|^{2}=\int_{\left\{1<u_{n} \leq k\right\}}\left|\nabla u_{n}\right|^{2} \\
& \geq \int_{\left\{\left|\nabla u_{n}\right|>t, 1<u_{n} \leq k\right\}}\left|\nabla u_{n}\right|^{2} \geq t^{2} m\left(\left\{\left|\nabla u_{n}\right|>t, 1<u_{n} \leq k\right\}\right),
\end{aligned}
$$

$$
m\left(\left\{\left|\nabla u_{n}\right|>t, 1<u_{n} \leq k\right\}\right) \leq \frac{C k}{t^{2}} \quad \forall k \geq 1 .
$$

By keeping track of the dependence on $k$ in the proof of Lemma 2.7 one readily realize that

$$
\int_{\Omega}\left|\nabla T_{k}^{\frac{\gamma+1}{2}}\left(u_{n}\right)\right|^{2} \leq C k^{\gamma}, \text { for any } k>1
$$

which, reasoning as in the proof of Lemma 2.5, gives

$$
m\left(\left\{u_{n}>k\right\}\right) \leq \frac{C}{k^{\frac{N}{N-2}}} \quad \forall k \geq 1 .
$$


In order to conclude we take again $k=t^{\frac{N-2}{N-1}}$ and we get

$$
m\left(\left\{\left|\nabla u_{n}\right|>t, u_{n}>1\right\}\right) \leq \frac{C}{t^{\frac{N}{N-1}}} \quad \forall t \geq 1
$$

and this proves (2.13).

Step 2. $T_{1}\left(u_{n}\right)$ is bounded in $H_{l o c}^{1}(\Omega)$.

We need to investigate the behavior of $u_{n}$ for small values (namely $u_{n} \leq 1$ ). We want to prove that for every $\omega \subset \subset \Omega$

$$
\int_{\omega}\left|\nabla T_{1}\left(u_{n}\right)\right|^{2} \leq C
$$

We have already proved that $u_{n} \geq c_{\omega}$ on $\omega$. We take $T_{1}^{\gamma}\left(u_{n}\right)$ as a test function in (2.8) in order to obtain

$$
\int_{\Omega} A(x) \nabla u_{n} \cdot \nabla T_{1}\left(u_{n}\right) T_{1}^{\gamma-1}\left(u_{n}\right)=\int_{\Omega} \frac{f_{n} T_{1}^{\gamma}\left(u_{n}\right)}{\left(u_{n}+\frac{1}{n}\right)^{\gamma}}+\int_{\Omega} \mu_{n} T_{1}^{\gamma}\left(u_{n}\right) \leq C .
$$

Now observe that

$$
\int_{\Omega} A(x) \nabla u_{n} \cdot \nabla T_{1}\left(u_{n}\right) T_{1}^{\gamma-1}\left(u_{n}\right) \geq \alpha \int_{\Omega}\left|\nabla T_{1}\left(u_{n}\right)\right|^{2} T_{1}^{\gamma-1}\left(u_{n}\right) \geq \alpha c_{\omega}^{\gamma-1} \int_{\omega}\left|\nabla T_{1}\left(u_{n}\right)\right|^{2} .
$$

Combining (2.16) and (2.17) we get (2.15).

The proof of Lemma 2.8 is complete as $u_{n}=T_{1}\left(u_{n}\right)+G_{1}\left(u_{n}\right)$, then $u_{n}$ is bounded in $W_{l o c}^{1, q}(\Omega)$ for every $q<\frac{N}{N-1}$.

We can finally state and prove the following existence result.

Theorem 2.9. Let $\gamma>1$. Then there exists a weak solution $u$ of (2.4) in $W_{\text {loc }}^{1, q}(\Omega)$ for every $q<\frac{N}{N-1}$.

Proof. Thanks to Lemma 2.7 and Lemma 2.8 above the proof of Theorem 2.9 is just a straightforward readaptation of the one of Theorem 2.6 .

2.3. Regularity of the solution. In this section we show how the solutions of problem (2.4) obtained in the previous sections increase their summability depending on the summability of the data.

We have the following

Theorem 2.10. Let $f \in L^{m}(\Omega)$ and let $\mu \in L^{r}(\Omega)$. Then there exists a solution u to (2.4) such that:

(i) if $m, r>\frac{N}{2}$, then $u \in L^{\infty}(\Omega)$,

(ii) if $1 \leq m<\frac{N}{2}, r>\frac{N}{2}$, then $u \in L^{\frac{N m(\gamma+1)}{N-2 m}}(\Omega)$,

(iii) if $m>\frac{N}{2}, 1<r<\frac{N}{2}$, then $u \in L^{r^{* *}}(\Omega)$,

(iv) if $1 \leq m<\frac{N}{2}, 1<r<\frac{N}{2}$, then $u \in L^{q}(\Omega)$, where $q=\min \left(\frac{N m(\gamma+1)}{N-2 m}, r^{* *}\right)$.

Proof. Let $u_{n}$ be the sequence of approximating solutions to problem (2.4) introduced in (2.8). Let $w_{n}$ be the sequence of solutions of

$$
\begin{cases}-\operatorname{div}\left(A(x) \nabla w_{n}\right)=\mu_{n} & \text { in } \Omega, \\ w_{n}=0 & \text { on } \partial \Omega,\end{cases}
$$

and let $v_{n}$ be the solutions of

$$
\begin{cases}-\operatorname{div}\left(A(x) \nabla v_{n}\right)=\frac{f_{n}(x)}{\left(v_{n}+\frac{1}{n}\right)^{\gamma}} & \text { in } \Omega, \\ v_{n}=0 & \text { on } \partial \Omega,\end{cases}
$$

where $f_{n}, \mu_{n}$ are the truncation at level $n$ of both $f$ and $\mu$.

Let us define $z_{n}=w_{n}+v_{n}$, thus we have

$$
-\operatorname{div}\left(A(x) \nabla z_{n}\right)=\frac{f_{n}(x)}{\left(v_{n}+\frac{1}{n}\right)^{\gamma}}+\mu_{n} \geq \frac{f_{n}(x)}{\left(z_{n}+\frac{1}{n}\right)^{\gamma}}+\mu_{n} .
$$


Taking $\left(z_{n}-u_{n}\right)^{-}$as test function in the formulation both (2.20) and (2.8), and taking the difference between the two we obtain

$$
\int_{\Omega} A(x) \nabla\left(z_{n}-u_{n}\right) \cdot \nabla\left(z_{n}-u_{n}\right)^{-} \geq \int_{\Omega}\left(\frac{f_{n}(x)}{\left(z_{n}+\frac{1}{n}\right)^{\gamma}}-\frac{f_{n}(x)}{\left(u_{n}+\frac{1}{n}\right)^{\gamma}}\right)\left(z_{n}-u_{n}\right)^{-} \geq 0,
$$

that implies

So we have

$$
\int_{\Omega}\left|\nabla\left(z_{n}-u_{n}\right)^{-}\right|^{2} \leq 0
$$

$$
u_{n} \leq z_{n}=w_{n}+v_{n}
$$

Passing to the limit in $n$ we then have that the summability of the solution $u$ can not be worse than $q$, where $q$ is the minimum between the summabilities of $w$ and $v$ that are investigated, respectively, in [28] and [8]. To show the optimality of this result what is left is the proof that

$$
w_{n} \leq u_{n}, \text { and } v_{n} \leq u_{n}
$$

The previous bounds can be found, reasoning as before, taking $\left(u_{n}-w_{n}\right)^{-}$as test functions in the difference between (2.8) and (2.18), and taking $\left(u_{n}-v_{n}\right)^{-}$, as test functions in the difference between (2.8) and (2.19). This completes the proof.

Remark 2.11. In Theorem 2.10 we have explicitly omitted the case where $r=1$. Let us observe that, according with the Stampacchia regularity result in [28, the statement holds true also in this case provided we substitute $1^{* *}\left(=\frac{N}{N-2}\right)$ with $1^{* *}-\epsilon$, where $\epsilon$ is any strictly positive fixed number.

2.4. Further remarks in a model case. In this section we consider a less general case, namely $A \equiv I$ and $\gamma<1$; we will show that something more can be said on suitable solutions to (2.4) in this Lazer-McKenna type model case.

We consider $\Omega$ to be a bounded open subset of $\mathbb{R}^{N}(N \geq 2)$ with boundary $\partial \Omega$ of class $C^{2+\alpha}$ for some $0<\alpha<1$. We consider the following semilinear elliptic problem

$$
\begin{cases}-\Delta u=\frac{f(x)}{u^{\gamma}}+\mu & \text { in } \Omega \\ u=0 & \text { on } \partial \Omega\end{cases}
$$

where $0<\gamma<1$, and $\mu$ is a nonnegative bounded Radon measure on $\Omega$.

Concerning the data $f$, for a fixed $\delta>0$, let

$$
\Omega_{\delta}:=\{x \in \Omega: \operatorname{dist}(x, \partial \Omega)<\delta\},
$$

and consider $f \in L^{1}(\Omega) \cap L^{\infty}\left(\Omega_{\delta}\right)$ such that $f>0$ a.e. in $\Omega$. Here, with a little abuse of notation, we mean that $f$ is a purely $L^{1}$ function defined on $\Omega$ that is essentially bounded in a neighborhood of $\partial \Omega$. These assumptions can be regarded as a suitable relaxation of the assumptions in [23].

We shall prove existence and uniqueness of a nonnegative weak solution to problem (2.21). Observe that, to this aim, the restriction to the case of the laplacian is not only technical. Also in the nonsingular case, uniqueness of distributional solutions for problems as (1.1) fails in general (see [27] and [6] for further results in this direction).

As we will see our argument is strongly based on the fact that, in this case, a suitable solution to (2.21) can be found satisfying a further regularity property, namely the integrability of the lower order singular term of the equation. This fact is in general false if $\gamma \geq 1$ even in the case $\mu \equiv 0$. This takes us to consider another notion of solution that is strictly related to the integrability of the singular term.

Here is the notion of solution we will consider.

Definition 2.12. A (weak) solution to problem (2.21) is a function $u \in L^{1}(\Omega)$ such that $u>0$ a.e. in $\Omega$, $f u^{-\gamma} \in L^{1}(\Omega)$, and

$$
-\int_{\Omega} u \Delta \varphi=\int_{\Omega} \frac{f \varphi}{u^{\gamma}}+\int_{\Omega} \varphi d \mu, \quad \forall \varphi \in C_{0}^{2}(\bar{\Omega}) .
$$


Remark 2.13. We recall that by $C_{0}^{2}(\bar{\Omega})$ we mean functions in $\varphi \in C^{2}(\bar{\Omega})$ with $\varphi=0$ on $\partial \Omega$. With this choice of test functions all terms in (2.22) are well defined and the boundary condition $u=0$ on $\partial \Omega$ is contained in (2.22). Also observe that, due to the uniqueness result we will prove, a solution $u$ in the above sense will coincide with a solution in the sense of Definition 2.5 provided $f u^{-\gamma} \in L^{1}(\Omega)$.

We will prove the following

Theorem 2.14. Let $f$ be an a.e. positive function in $L^{1}(\Omega) \cap L^{\infty}\left(\Omega_{\delta}\right)$, for some $\delta>0$. Then there exists a unique solution for problem (2.21) in the sense of Definition [2.12.

In order to prove Theorem 2.14 we need to prove first that a solution in the sense of Definition 2.12 does exist. This can be easily checked by sub and supersolutions method. We start by define the concept of sub and supersolutions for problem (2.21).

Definition 2.15. A function $\underline{u}$ is a subsolution for (2.21) if $\underline{u} \in L^{1}(\Omega), \underline{u}>0$ in $\Omega, f \underline{u}^{-\gamma} \in L^{1}(\Omega)$, and

$$
-\int_{\Omega} \underline{u} \Delta \varphi \leq \int_{\Omega} \frac{f \varphi}{\underline{u}^{\gamma}}+\int_{\Omega} \varphi d \mu, \quad \forall \varphi \in C_{0}^{2}(\bar{\Omega}), \varphi \geq 0 .
$$

Analogously, we say that $\bar{u}$ is a supersolution for problem (2.21) if $\bar{u} \in L^{1}(\Omega), \bar{u}>0$ in $\Omega, f \bar{u}^{-\gamma} \in L^{1}(\Omega)$, and (2.23) is satisfied with the opposite inequality sign (i.e. $\geq$ ).

We are ready to state the result that is the basis of the method of sub and supersolutions for problem (2.21). The proof is suitable re-adaptation of an argument in [25] and we will only sketch it.

Theorem 2.16. If problem (2.21) has a subsolution $\underline{u}$ and supersolution $\bar{u}$ with $\underline{u} \leq \bar{u}$ in $\Omega$, then there exists a solution $u$ to (2.21) (in the sense of Definition 2.12) such that $\underline{u} \leq u \leq \bar{u}$.

Sketch of the proof. We first considered a truncated problem with a modified nonlinearity $\tilde{g}(x, u)$. The goal consists in proving that the solution of this truncated problem turns out to solve problem (2.21).

We define $\tilde{g}: \Omega \times \mathbb{R} \rightarrow \mathbb{R}$ as

$$
\tilde{g}(x, t)= \begin{cases}f(x) \underline{u}(x)^{-\gamma} & \text { if } t<\underline{u}(x), \\ f(x) t^{-\gamma} & \text { if } \underline{u}(x) \leq t \leq \bar{u}(x), \\ f(x) \bar{u}(x)^{-\gamma} & \text { if } t>\bar{u}(x) .\end{cases}
$$

Notice that by definition of sub and supersolution both $f \underline{u}^{-\gamma}$ and $f \bar{u}^{-\gamma}$ belong to $L^{1}(\Omega)$. Moreover, $\underline{u}>0$ so that, $\tilde{g}$ is well defined a.e. on $\Omega$ and for every fixed $v \in L^{1}(\Omega)$ we have that $\tilde{g}(x, v(x)) \in L^{1}(\Omega)$.

First of all, if $u$ satisfies

$$
\begin{cases}-\Delta u=\tilde{g}(x, u)+\mu & \text { in } \Omega, \\ u=0 & \text { on } \partial \Omega,\end{cases}
$$

then $\underline{u} \leq u \leq \bar{u}$. Thus $\tilde{g}(\cdot, u)=g(u), f u^{-\gamma} \in L^{1}(\Omega)$, and $u$ is a solution to (2.21). This fact can be checked by mean of Kato's inequality up to the boundary (see [26, Lemma 6.11]) applied to the function $u-\bar{u}$. The proof is complete if we show that a solution to problem (2.24) does exist. Let us define

$$
\begin{gathered}
G: L^{1}(\Omega) \rightarrow L^{1}(\Omega), \\
v \rightarrow u .
\end{gathered}
$$

This map assigns to every $v \in L^{1}(\Omega)$ the solution $u$ to the following linear problem

$$
\begin{cases}-\Delta u=\tilde{g}(x, v)+\mu & \text { in } \Omega \\ u=0 & \text { on } \partial \Omega\end{cases}
$$

Standard Schauder fixed point theorem applies and one can easily verifies that a solution $u$ to (2.24) does exist. In view of what we said before, this concludes the proof of Theorem 2.16 .

We are now in the position to prove Theorem 2.14 . 
Proof of Theorem 2.14. As we have $f u^{-\gamma} \in L^{1}(\Omega)$, then the uniqueness is an easy consequence of Proposition 4.B.3 in [12]. In fact, if $u_{1}$ and $u_{2}$ are two solutions of (2.21) in the sense of Definition 2.12 with data $\mu_{1}$ and $\mu_{2}$ respectively, then one has

$$
\int_{\Omega} f\left|\frac{1}{u_{2}^{\gamma}}-\frac{1}{u_{1}^{\gamma}}\right| \leq \int_{\Omega} d\left|\mu_{1}-\mu_{2}\right|
$$

from which uniqueness is deduced as $f>0$ in $\Omega$.

In order to prove existence we apply Theorem 2.16. We need to find both a subsolution and a supersolution to problem (2.21) in the sense of Definition 2.15,

We first look for a subsolution. Let us consider the following problem

$$
\begin{cases}-\Delta v=\frac{f(x)}{v^{\gamma}} & \text { in } \Omega, \\ v=0 & \text { on } \partial \Omega .\end{cases}
$$

It is proved in $\left[8\right.$ the existence of a solution $v \in L^{1}(\Omega)$ to problem (2.25). We need a sharp estimate near the boundary for $v$. We consider the following approximating problems

$$
\begin{cases}-\Delta v_{n}=\frac{f_{n}(x)}{\left(v_{n}+\frac{1}{n}\right)^{\gamma}} & \text { in } \Omega, \\ v_{n}=0 & \text { on } \partial \Omega,\end{cases}
$$

where $f_{n}$ is the truncation at level $n$ of $f$ (i.e. $f_{n}=T_{n}(f)$ ). It was proven in 8 that the nondecreasing sequence $v_{n}$ converges to a solution of problem (2.25). By the linear theory, the sequence $v_{n}$ belongs to $L^{\infty}(\Omega)$. Also observe that the term $\frac{f_{1}}{\left(v_{1}+1\right)^{\gamma}}$ belongs to $L^{\infty}(\Omega)$ so that we can apply Lemma 3.2 in [1] in order to obtain that for a.e. $x$ in $\Omega$

$$
\frac{v_{1}(x)}{d(x)} \geq C \int_{\Omega} \frac{d(y) f_{1}(y)}{\left\|v_{1}\right\|_{L^{\infty}(\Omega)}+1} d y \geq C>0,
$$

where $d(x):=d(x, \partial \Omega)$ is the distance function of $x$ from $\partial \Omega$. Thus, we have

$$
v(x) \geq v_{1}(x) \geq C_{1} d(x) \text {, a.e. on } \Omega .
$$

Therefore, as $f \in L^{\infty}\left(\Omega_{\delta}\right)$, then $f v^{-\gamma} \leq f d(x)^{-\gamma}$ is integrable in $\Omega$ for every $\gamma<1$. Thus we have that $v>0$ in $\Omega, f v^{-\gamma} \in L^{1}(\Omega)$, and

$$
-\int_{\Omega} v \Delta \varphi=\int_{\Omega} \frac{f \varphi}{v^{\gamma}}, \quad \forall \varphi \in C_{0}^{2}(\bar{\Omega}) .
$$

Since $\mu$ is a nonnegative Radon measure we clearly have

$$
-\int_{\Omega} v \Delta \varphi \leq \int_{\Omega} \frac{f \varphi}{v^{\gamma}}+\int_{\Omega} \varphi d \mu, \quad \forall \varphi \in C_{0}^{2}(\bar{\Omega}), \varphi \geq 0,
$$

that is, $v$ is a subsolution to the problem (2.21).

We now look for a supersolution of problem (2.21). Let $w$ be the solution of

$$
\begin{cases}-\Delta w=\mu & \text { in } \Omega, \\ w=0 & \text { on } \partial \Omega .\end{cases}
$$

The existence of a positive solution to the problem (2.26) is classical (see for instance [28] where the solution is obtained by duality in a more general framework).

Let us define $z:=w+v$ where $v$ is again the solution to (2.25). We have

$$
-\int_{\Omega} z \Delta \varphi=-\int_{\Omega} w \Delta \varphi-\int_{\Omega} v \Delta \varphi=\int_{\Omega} \varphi d \mu+\int_{\Omega} \frac{f \varphi}{v^{\gamma}}, \quad \forall \varphi \in C_{0}^{2}(\bar{\Omega}) .
$$

Recalling that $w$ is nonnegative, then we have $z^{\gamma} \geq v^{\gamma}>0$. Thus, we can say

$$
\int_{\Omega} \varphi d \mu+\int_{\Omega} \frac{\varphi}{v^{\gamma}} \geq \int_{\Omega} \varphi d \mu+\int_{\Omega} \frac{f \varphi}{z^{\gamma}}, \quad \forall \varphi \in C_{0}^{2}(\bar{\Omega}), \varphi \geq 0
$$


that is, $z$ is a positive function in $L^{1}(\Omega)$ such that $z^{-\gamma} \leq v^{-\gamma} \in L^{1}(\Omega)$ and

$$
-\int_{\Omega} z \Delta \varphi \geq \int_{\Omega} \frac{f \varphi}{z^{\gamma}}+\int_{\Omega} \varphi d \mu, \quad \forall \varphi \in C_{0}^{2}(\bar{\Omega}), \varphi \geq 0 .
$$

Thus, $z$ is a supersolution to (2.21). We can then apply Theorem 2.16 to conclude that there exists a solution $u$ to problem (2.21) in the sense of Definition 2.12,

Remark 2.17. We stress that strict positivity of $f$ in $\Omega$ is only used to deduce uniqueness of a solution. For a purely nonnegative $f$, then the existence of a solution can be deduced exactly with the same argument.

\section{NONLINEAR PRINCIPAL PART}

In this last section we show how the existence results proved before can be extended to the case of a nonlinear principal part. For a given real number $p$ with $2-\frac{1}{N}<p<N$, let $a: \Omega \times \mathbb{R}^{N} \rightarrow \mathbb{R}^{N}$ be a Carathéodory function such that there exist $\alpha, \beta>0$ with

$$
\begin{gathered}
\left(a(x, \xi)-a\left(x, \xi^{*}\right)\right) \cdot\left(\xi-\xi^{*}\right)>0, \\
a(x, \xi) \cdot \xi>\alpha|\xi|^{p} \\
a(x, \xi) \leq \beta\left(c(x)+|\xi|^{p-1}\right),
\end{gathered}
$$

for every $\xi, \xi^{*} \in \mathbb{R}^{N}$ such that $\xi \neq \xi^{*}$, for almost every $x$ in $\Omega$, and $c(x)$ belongs to $L^{p^{\prime}}(\Omega)$. The operator

$$
-\operatorname{div}(a(x, \nabla u))
$$

is a classical Leray-Lions type operator which maps continuously $W_{0}^{1, p}(\Omega)$ into its dual $W^{-1, p^{\prime}}(\Omega)$ whose simplest model is the $p$-laplacian (i.e. $a(x, \xi)=|\xi|^{p-2} \xi$ ).

We will prove existence of a nonnegative weak solution to the following problem

$$
\begin{cases}-\operatorname{div}(a(x, \nabla u))=\frac{f(x)}{u^{\gamma}}+\mu & \text { in } \Omega, \\ u=0 & \text { on } \partial \Omega,\end{cases}
$$

where $\Omega$ is an open bounded subset of $\mathbb{R}^{N}, N \geq 2, \gamma>0, f$ is a nonnegative function which belongs to $L^{1}(\Omega)$ and $\mu$ is a nonnegative bounded Radon measure.

The bound from below for $p$ is a classical technical assumption that guarantees, even for nonsmooth data, that the gradient of the solutions will belong at least to $\left(L_{l o c}^{1}(\Omega)\right)^{N}$.

Here is the suitable definition for weak solutions to (3.30).

Definition 3.1. If $\gamma \leq 1$, a weak solution to problem (3.30) is a function $u \in W_{0}^{1,1}(\Omega)$ such that

$$
\forall \omega \subset \subset \Omega \exists c_{\omega}: u \geq c_{\omega}>0,
$$

and such that

$$
\int_{\Omega} a(x, \nabla u) \cdot \nabla \varphi=\int_{\Omega} \frac{f \varphi}{u^{\gamma}}+\int_{\Omega} \varphi d \mu, \quad \forall \varphi \in C_{c}^{1}(\Omega) .
$$

If $\gamma>1$ a weak solution for problem (3.30) is a function $u \in W_{l o c}^{1,1}(\Omega)$ such that (3.31) and (3.32) are satisfied and $T_{k}^{\frac{\gamma-1+p}{p}}(u)$ belongs to $W_{0}^{1, p}(\Omega)$ for every fixed $k>0$.

Theorem 3.2. Let $\gamma>0$. Then there exists a weak solution $u$ to (3.30) in the sense of Definition 3.1. Moreover:

(i) if $\gamma \leq 1$, then $u \in W_{0}^{1, q}(\Omega)$ for every $q<\frac{N(p-1)}{N-1}$,

(ii) if $\gamma>1$, then $u \in W_{l o c}^{1, q}(\Omega)$ for every $q<\frac{N(p-1)}{N-1}$.

Proof. The proof of Theorem 3.2 strictly follows the main steps of the previous section. We will then sketch it by enlightening the main differences. Estimates will essentially involve ellipticity and so they will be formally very similar to the ones in Section 2 The main issue in this case will be to pass to the limit in the principal part of the approximating solutions for which we will need to prove the almost everywhere convergence of the gradients that will be based on monotonicity arguments relying on (3.27). 
Step 1. Existence for the approximating problems. Let us consider the following problem

$$
\begin{cases}-\operatorname{div}\left(a\left(x, \nabla u_{n}\right)\right)=\frac{f_{n}}{\left(u_{n}+\frac{1}{n}\right)^{\gamma}}+\mu_{n} & \text { in } \Omega \\ u_{n}=0 & \text { on } \partial \Omega\end{cases}
$$

where, as before, $f_{n}$ is the truncation at level $n$ of $f$ and $\mu_{n}$ is a sequence of smooth functions bounded in $L^{1}(\Omega)$ and converging weakly to $\mu$ in the sense of the measures.

The proof of existence of a weak solution of problem (3.33) for every fixed $n \in \mathbb{N}$ is formally identical to the one in Lemma 2.3. We define the operator

$$
G: L^{p}(\Omega) \rightarrow L^{p}(\Omega)
$$

that assigns to every $v \in L^{p}(\Omega)$ the solution $w$ to the following problem

$$
\begin{cases}-\operatorname{div}(a(x, \nabla w))=\frac{f_{n}}{\left(|v|+\frac{1}{n}\right)^{\gamma}}+\mu_{n} & \text { in } \Omega, \\ w=0 & \text { on } \partial \Omega .\end{cases}
$$

A straightforward re-adaptation of the proof of Lemma 2.3 allows us to prove both that the ball of radius $C^{\prime}\left(n^{\gamma+1}+C(n)\right)$ is invariant for $G$ and the set $G\left(L^{p}(\Omega)\right)$ is relatively compact in $L^{p}(\Omega)$. Concerning the continuity of $G$ we use monotonicity. Let us choose a sequence $v_{k}$ that converges to $v$ in $L^{p}(\Omega)$, then we need to prove that $G\left(v_{k}\right)$ converges to $G(v)$ in $L^{p}(\Omega)$. By compactness we already know that the sequence $G\left(v_{k}\right)$ converges to some function $w$ in $L^{p}(\Omega)$. We only need to prove that $w=G(v)$. This means that we need to pass to the limit with respect to $k$ in the following weak formulation

$$
\int_{\Omega} a\left(x, \nabla w_{k}\right) \cdot \nabla \varphi=\int_{\Omega} \frac{f_{n} \varphi}{\left(\left|v_{k}\right|+\frac{1}{n}\right)^{\gamma}}+\int_{\Omega} \mu_{n} \varphi,
$$

where $\varphi \in W_{0}^{1, p}(\Omega)$ and $w_{k}=G\left(v_{k}\right)$.

All terms but the one on the left hand side of (3.34) pass to the limit. Concerning the term on the left hand side we need to check the almost everywhere convergence of the gradients of $w_{k}$.

We take $w_{k}-w$ as test function in the weak formulation of (3.33)

$$
\begin{array}{r}
\int_{\Omega}\left(a\left(x, \nabla w_{k}\right)-a(x, \nabla w)\right) \cdot \nabla\left(w_{k}-w\right)=\int_{\Omega} \frac{f_{n}\left(w_{k}-w\right)}{\left(\left|v_{k}\right|+\frac{1}{n}\right)^{\gamma}} \\
+\int_{\Omega} \mu_{n}\left(w_{k}-w\right)-\int_{\Omega} a(x, \nabla w) \cdot \nabla\left(w_{k}-w\right) .
\end{array}
$$

Since $w_{k}$ converges to $w$ in $L^{p}(\Omega)$, then the first and the second term on the right hand side of (3.35) tends to zero when $k$ tends to infinity. Also the third term tends to zero since, by classical theory (see for instance [24]), we have that $w_{k}-w$ weakly converges to zero in $W_{0}^{1, p}(\Omega)$ and $a(x, \nabla w)$ is a fixed function in $\left(L^{p^{\prime}}(\Omega)\right)^{N}$. This means that the term on the left hand side of (3.35) tends to zero so that we can apply Lemma 5 in 9 , to obtain that $\nabla w_{k}$ converges almost everywhere to $\nabla w$. This means that $w=G(v)$. We can then apply Schauder fixed point theorem and maximum principle in order to get the existence of a nonnegative solution in $W_{0}^{1, p}(\Omega)$ for problem (3.33). Moreover, by classical regularity theory (see for instance [9]), $u_{n}$ belongs to $L^{\infty}(\Omega)$.

Step 2. Local uniform bound from below. Here we show that $u_{n}$ is bounded from below on the compact subsets of $\Omega$. In particular, we want to check that the sequence $u_{n}$ is such that for every $\omega \subset \subset \Omega$ there exists $c_{\omega}$ (not depending on $n$ ) such that

$$
u_{n}(x) \geq c_{\omega}>0 \text {, for a.e. } x \text { in } \omega \text {, for every } \mathrm{n} \text { in } \mathbb{N} \text {. }
$$

We consider the sequence of problems

$$
\begin{cases}-\operatorname{div}\left(a\left(x, \nabla v_{n}\right)\right)=\frac{f_{n}}{\left(v_{n}+\frac{1}{n}\right)^{\gamma}} & \text { in } \Omega \\ v_{n}=0 & \text { on } \partial \Omega .\end{cases}
$$


It was proved in [19] the existence of a weak solution for (3.37) such that

$$
\forall \omega \subset \subset \Omega \exists c_{\omega}: v_{n} \geq c_{\omega}>0,
$$

for almost every $x$ in $\omega$ and where $c_{\omega}$ is indipendent on $n$.

Thus, taking $\left(u_{n}-v_{n}\right)^{-}$as test function in the difference between (3.33) and (3.37), we obtain

$$
\begin{aligned}
& \int_{\Omega}\left(a\left(x, \nabla u_{n}\right)-a\left(x, \nabla v_{n}\right)\right) \cdot \nabla\left(u_{n}-v_{n}\right)^{-} \\
& =\int_{\Omega}\left(\frac{f_{n}(x)}{\left(u_{n}+\frac{1}{n}\right)^{\gamma}}-\frac{f_{n}(x)}{\left(v_{n}+\frac{1}{n}\right)^{\gamma}}\right)\left(u_{n}-v_{n}\right)^{-}+\int_{\Omega} \mu_{n}\left(u_{n}-v_{n}\right)^{-} \geq 0,
\end{aligned}
$$

so that, by (3.27)

$$
0 \geq-\int_{\left\{u_{n} \leq v_{n}\right\}}\left(a\left(x, \nabla u_{n}\right)-a\left(x, \nabla v_{n}\right)\right) \cdot \nabla\left(u_{n}-v_{n}\right) \geq 0 .
$$

This implies, by monotonicity, that

$$
\nabla\left(u_{n}-v_{n}\right)^{-}=\nabla\left(u_{n}-v_{n}\right) \chi_{\left\{u_{n} \leq v_{n}\right\}}=0,
$$

so that,

$$
v_{n} \leq u_{n}
$$

and so

$$
\forall \omega \subset \subset \Omega \exists c_{\omega}: u_{n} \geq v_{n} \geq c_{\omega}>0,
$$

for almost every $x$ in $\omega$.

Step 3. Estimates on the approximating solutions. Here we look for some estimates on $u_{n}$ in some Sobolev spaces. As in the semilinear case these estimates will depend on the value of $\gamma$.

First of all we introduce an auxiliary function that will be useful for our purpose

$$
S_{k}(s)= \begin{cases}1 & s>k+1 \\ s-k & k<s \leq k+1 \\ 0 & 0 \leq s \leq k\end{cases}
$$

We observe that if $s \geq 0$ then $S_{k}(s) \leq s$.

The case $\gamma \leq 1$. We will prove that $u_{n}$ is bounded in $W_{0}^{1, q}(\Omega)$ for every $q<\frac{N(p-1)}{N-1}$.

As in the semilinear case we first observe that the truncations of the approximating solutions are bounded in the energy space $W_{0}^{1, p}(\Omega)$. In fact, we take $\left(T_{k}\left(u_{n}\right)+\epsilon\right)^{\gamma}-\epsilon^{\gamma}$ as test function in the weak formulation of (3.33) where $\epsilon$ is a fixed number strictly smaller than $\frac{1}{n}$ (the case $\gamma=1$ implies the obvious simplifications). Reasoning as in the proof of Lemma 2.5 we readily obtain

$$
\int_{\Omega}\left|\nabla T_{k}\left(u_{n}\right)\right|^{p} \leq C\left(k^{1-\gamma}+k\right) .
$$

In particular

$$
\int_{\Omega}\left|\nabla T_{1}\left(u_{n}\right)\right|^{p} \leq C
$$

In order to get an estimate for $u_{n}$ in $W_{0}^{1, q}(\Omega)$ for every $q<\frac{N(p-1)}{N-1}$ it suffices to look for this bound on $G_{1}\left(u_{n}\right)$.

We take $T_{k}\left(G_{1}\left(u_{n}\right)\right)$ as test function in the weak formulation of (3.33) for $k>1$. Then we have

$$
\int_{\Omega} a\left(x, \nabla u_{n}\right) \cdot \nabla T_{k}\left(G_{1}\left(u_{n}\right)\right) \leq \int_{\Omega} \frac{f_{n}\left[\left(T_{k}\left(G_{1}\left(u_{n}\right)\right)\right]\right.}{\left(u_{n}+\frac{1}{n}\right)^{\gamma}}+\int_{\Omega} \mu_{n} T_{k}\left(G_{1}\left(u_{n}\right)\right) \leq C k,
$$

and also

$$
\int_{\Omega} a\left(x, \nabla u_{n}\right) \cdot \nabla T_{k}\left(G_{1}\left(u_{n}\right)\right)=\int_{\Omega} a\left(x, \nabla T_{k}\left(G_{1}\left(u_{n}\right)\right)\right) \cdot \nabla T_{k}\left(G_{1}\left(u_{n}\right)\right) \geq \alpha \int_{\Omega}\left|\nabla T_{k}\left(G_{1}\left(u_{n}\right)\right)\right|^{p},
$$


so that we obtain

$$
\int_{\Omega}\left|\nabla T_{k}\left(G_{1}\left(u_{n}\right)\right)\right|^{p} \leq C k
$$

Now we take $S_{k}\left(G_{1}\left(u_{n}\right)\right)$ as test function again in the weak formulation of (3.33), so that

$$
\int_{\Omega} a\left(x, \nabla u_{n}\right) \cdot \nabla G_{1}\left(u_{n}\right) S_{k}^{\prime}\left(G_{1}\left(u_{n}\right)\right) \leq C
$$

and again

and finally

$$
\begin{aligned}
& \int_{\Omega} a\left(x, \nabla u_{n}\right) \cdot \nabla G_{1}\left(u_{n}\right) S_{k}^{\prime}\left(G_{1}\left(u_{n}\right)\right) \\
& =\int_{\left\{k \leq G_{1}\left(u_{n}\right) \leq k+1\right\}} a\left(x, \nabla G_{1}\left(u_{n}\right)\right) \cdot \nabla G_{1}\left(u_{n}\right) \geq \int_{\left\{k \leq G_{1}\left(u_{n}\right) \leq k+1\right\}}\left|\nabla G_{1}\left(u_{n}\right)\right|^{p},
\end{aligned}
$$

$$
\int_{\left\{k \leq G_{1}\left(u_{n}\right) \leq k+1\right\}}\left|\nabla G_{1}\left(u_{n}\right)\right|^{p} \leq C .
$$

Thanks to estimates (3.38) and (3.39) we can proceed as in Section II. 4 of [7] in order to obtain that $G_{1}\left(u_{n}\right)$ is bounded in $W_{0}^{1, q}(\Omega)$ for every $q<\frac{N(p-1)}{N-1}$. Thus we can conclude that $u_{n}$ is bounded in $W_{0}^{1, q}(\Omega)$ for every $q<\frac{N(p-1)}{N-1}$.

The case $\gamma>1$. As in the semilinear case, here we only look for local estimates. First of all we observe that the (global) bound on $G_{1}\left(u_{n}\right)$ in $W_{0}^{1, q}(\Omega)$ for every $q<\frac{N(p-1)}{N-1}$ follows exactly as in the the case $\gamma \leq 1$.

What is left is the proof that $T_{k}\left(u_{n}\right)$ is bounded in $W_{l o c}^{1, p}(\Omega)$ for every $k>0$. We take $\varphi=T_{k}^{\gamma}\left(u_{n}\right)$ as test function in the weak formulation of (3.33) in order to have

$$
\int_{\Omega} a\left(x, \nabla u_{n}\right) \cdot \nabla T_{k}\left(u_{n}\right) T_{k}^{\gamma-1}\left(u_{n}\right) \leq C+C k^{\gamma}
$$

so that using (3.36) we obtain

$$
\int_{\Omega} a\left(x, \nabla u_{n}\right) \cdot \nabla T_{k}\left(u_{n}\right) T_{k}^{\gamma-1}\left(u_{n}\right) \geq \alpha \int_{\Omega}\left|\nabla T_{k}\left(u_{n}\right)\right|^{p} T_{k}^{\gamma-1}\left(u_{n}\right) \geq C \int_{\omega}\left|\nabla T_{k}\left(u_{n}\right)\right|^{p},
$$

where $\omega$ is an arbitrary compact subset of $\Omega$. Thus, we have

$$
\int_{\omega}\left|\nabla T_{k}\left(u_{n}\right)\right|^{p} \leq C+C k^{\gamma}
$$

for every $\omega \subset \subset \Omega$ and every $k>0$.

Combining the estimates on both $G_{1}\left(u_{n}\right)$ and $T_{1}\left(u_{n}\right)$ we deduce that $u_{n}$ is uniformly bounded in $W_{l o c}^{1, q}(\Omega)$ for every $q<\frac{N(p-1)}{N-1}$.

In order to give sense to the function on the boundary of $\Omega$ we reason as before, we take $\varphi=T_{k}^{\gamma}\left(u_{n}\right)$ as test function in the weak formulation of (3.33) and we use ellipticity in order to get

$$
\int_{\Omega}\left|\nabla T_{k}^{\frac{\gamma-1+p}{p}}\left(u_{n}\right)\right|^{p} \leq C\left(1+k^{\gamma}\right) .
$$

Thanks to the estimates we proved here we readily deduce, by compact embeddings, the existence of a function $u$ such that (up to not relabeled subsequences) $u_{n}$ converges to $u$ a.e., strongly in $L^{1}(\Omega)$ and weakly in $W_{0}^{1, q}(\Omega)\left(W_{l o c}^{1, q}(\Omega)\right.$ if $\left.\gamma>1\right)$ for every $q<\frac{N(p-1)}{N-1}$. In particular $a\left(x, \nabla u_{n}\right)$ is bounded in $W_{l o c}^{1, q}(\Omega)$ for every $q<\frac{N}{N-1}$. So that in order to pass to the limit in (3.33) and to conclude the proof of Theorem 3.2 we only need to check the a.e. convergence of $\nabla u_{n}$ towards $\nabla u$.

Step 4. The a.e. convergence of the gradients. The a.e. convergence of $\nabla u_{n}$ towards $\nabla u$ follows in a standard way if we prove that $\nabla T_{k}\left(u_{n}\right)$ converges to $\nabla T_{k}(u)$ in $L_{l o c}^{q}(\Omega)$ for every $q<p$, for every $k>0$.

By Definition 2.29 and Remark 2.32 in [18] we know that $T_{k}\left(u_{n}\right)$ is such that

$$
-\operatorname{div}\left(a\left(x, \nabla T_{k}\left(u_{n}\right)\right)\right)=\left(\frac{f_{n}}{\left(\left|u_{n}\right|+\frac{1}{n}\right)^{\gamma}}+\mu_{n}\right) \chi_{\left\{\left|u_{n}\right| \leq k\right\}}+\lambda_{n, k} \quad \text { in } \Omega,
$$


where $\lambda_{k, n}$ is a nonnegative diffuse measure (i.e. it is an absolutely continuous measure with respect to the $H^{1}$-capacity) concentrated on the set $\left\{u_{n}=k\right\}$.

The first term on the right hand side of (3.40) is bounded in $L_{l o c}^{1}(\Omega)$.

To bound the second term we take $T_{k}^{\gamma}\left(u_{n}\right)$ as a test function in the weak formulation of (3.33) to have

$$
\int_{\Omega} a\left(x, \nabla u_{n}\right) \cdot \nabla T_{k}\left(u_{n}\right) T_{k}^{\gamma-1}\left(u_{n}\right) \leq C\|f\|_{L^{1}(\Omega)}+k^{\gamma}\left\|\mu_{n}\right\|_{L^{1}(\Omega)} .
$$

Now we take $T_{k}^{\gamma}\left(u_{n}\right)$ as a test function in the weak formulation of (3.40)

$$
\begin{aligned}
& \int_{\Omega} a\left(x, \nabla T_{k}\left(u_{n}\right)\right) \cdot \nabla T_{k}\left(u_{n}\right) T_{k}^{\gamma-1}\left(u_{n}\right) \\
& =\int_{\left\{\left|u_{n}\right| \leq k\right\}}\left(\frac{f_{n}}{\left(\left|u_{n}\right|+\frac{1}{n}\right)^{\gamma}}+\mu_{n}\right) T_{k}^{\gamma}\left(u_{n}\right)+k^{\gamma} \lambda_{n, k}(\Omega) .
\end{aligned}
$$

Since the first term on the right hand side of (3.42) is positive then the estimates (3.41) and (3.42) imply

$$
\lambda_{n, k}(\Omega) \leq \frac{C}{k^{\gamma}}
$$

so that $\lambda_{n, k}$ is uniformly bounded in as a measure with respect to $n$, for every fixed $k \geq 1$. Due to these bounds on the right hand side of (13.40) , as $T_{k}\left(u_{n}\right)$ is bounded in $W_{l o c}^{1, p}(\Omega)$, we can proceed as in the proof of Theorem 2.1 in [10]. In fact, one can obtain that

$$
\limsup _{n} \int_{\omega}\left(a\left(x, \nabla T_{k}\left(u_{n}\right)\right)-a\left(x, \nabla T_{k}(u)\right)\right) \cdot \nabla T_{h}\left(T_{k}\left(u_{n}\right)-T_{k}(u)\right) \leq C_{\omega, k} h
$$

for every $\omega \subset \subset \Omega$ and $h>0$. The previous estimate is known to imply (see again [10]) that $\nabla T_{k}\left(u_{n}\right)$ converges to $\nabla T_{k}(u)$ in $L_{l o c}^{q}(\Omega)$ for every $q<p$.

Remark 3.3. We point out that, for the sake of exposition, we assumed that the operator $a$ was chosen to be independent of $u$. Anyway one can easily realize that the same proof can be straightforwardly extended to more general Leray-Lions operator involving Carathéodory functions $a: \Omega \times \mathbb{R} \times \mathbb{R}^{N} \rightarrow \mathbb{R}^{N}$ such that

$$
\begin{gathered}
\left(a(x, s, \xi)-a\left(x, s, \xi^{*}\right)\right) \cdot\left(\xi-\xi^{*}\right)>0, \\
a(x, s, \xi) \cdot \xi>\alpha|\xi|^{p}, \\
a(x, s, \xi) \leq \beta\left(c(x)+|s|^{p-1}+|\xi|^{p-1}\right),
\end{gathered}
$$

for every $\xi, \xi^{*} \in \mathbb{R}^{N}$ such that $\xi \neq \xi^{*}$, for almost every $x$ in $\Omega, s \in \mathbb{R}, 0<\alpha \leq \beta$, and $c(x)$ in $L^{p^{\prime}}(\Omega)$.

Acknowledgement. The authors would like to thank the anonymous reviewers whose comments and suggestions helped to improve the final version of this manuscript. Also, the authors are partially supported by the Gruppo Nazionale per l'Analisi Matematica, la Probabilità e le loro Applicazioni (GNAMPA) of the Istituto Nazionale di Alta Matematica (INdAM).

\section{REFERENCES}

[1] D. Arcoya, J. Carmona, T. Leonori, P.J. Martínez-Aparicio, L. Orsina, F. Petitta, Existence and nonexistence of solutions for singular quadratic quasilinear equations. J. Diff. Equations 246 (10) (2009), 4006-4042.

[2] D. Arcoya, J. Carmona, P. J. Martínez-Aparicio, Bifurcation for quasilinear elliptic singular BVP. Comm. Partial Differential Equations 36 (4), (2011), 670-692.

[3] D. Arcoya, L. Moreno-Mérida, Multiplicity of solutions for a Dirichlet problem with a strongly singular nonlinearity. Nonlinear Analysis 95 (2014) 281-291.

[4] L. Boccardo, Dirichlet problems with singular and gradient quadratic lower order terms. ESAIM Control Optim. Calc. Var. 14 (3) (2008), 411-426.

[5] P. Benilan, L. Boccardo, T. Gallouët, R. Gariepy, M. Pierre, J. L. Vazquez, An $L^{1}$ theory of existence and uniqueness of nonlinear elliptic equations. Ann. Scuola Norm. Sup. Pisa 22 (1995), 240-273.

[6] L. Boccardo, J. Casado-Díaz, Some properties of solutions of some semilinear elliptic singular problems and applications to the G-convergence. Asymptotic Analysis 86 (2014), 1-15. 
[7] L. Boccardo, T. Gallouët, Non-linear elliptic and parabolic equations involving measure data. J. Funct. Anal. 87 (1989), $149-169$.

[8] L. Boccardo, L. Orsina, Semilinear elliptic equations with singular nonlinearities. Calc. Var. and PDEs 37 (3-4) (2010), $363-380$.

[9] L. Boccardo, F. Murat, J.P. Puel, Existence of bounded solutions for nonlinear unilateral problems. Ann. Mat. Pura Appl. 152 (1988), 183-196.

[10] L. Boccardo, F. Murat, Almost everywhere convergence of the gradients of solutions to elliptic and parabolic equations. Nonlinear Analysis 19 (6), (1992), 581-597.

[11] H. Brezis, X. Cabré, Some simple nonlinear PDE's without solutions. Bollettino dell'Unione Matematica Italiana Serie 8, 1-B (2) (1998), 223-262.

[12] H. Brezis, M. Marcus, A. C. Ponce, Nonlinear elliptic equations with measures revisited. Annals of Math. Studies, Princeton University Press NJ, 163, (2007), 55-110.

[13] A. Canino, Minimax methods for singular elliptic equations with an application to a jumping problem. J. Diff. Equations 221 (1), (2006), 210-223.

[14] A. Canino, M. Degiovanni, A variational approach to a class of singular semilinear elliptic equations. J. of Convex Analysis 11 (1), (2004), 147-162.

[15] A. Canino, M. Grandinetti, B. Sciunzi, Symmetry of solutions of some semilinear elliptic equations with singular nonlinearities. J. Diff. Equations 255 (12), (2013), 4437-4447.

[16] G. M. Coclite, M. M. Coclite, On a Dirichlet problem in bounded domains with singular nonlinearity. Discrete Contin. Dyn. Syst. 33 (11-12), (2013), 4923-4944.

[17] M. G. Crandall, P. H. Rabinowitz, L. Tartar, On a dirichlet problem with a singular nonlinearity. Comm. Part. Diff. Eq. 2 (2), (1977), 193-222.

[18] G. Dal Maso, F. Murat, L. Orsina, A. Prignet, Renormalized solutions of elliptic equations with general measure data. Annali della Scuola Normale Superiore di Pisa 28 (1999), 741-808.

[19] L. M. De Cave, Nonlinear elliptic equations with singular nonlinearities. Asymptotic Analysis 84 (2013), $181-195$.

[20] D. Giachetti, P. J. Martínez-Aparicio, F. Murat, Elliptic equations with mild singularities: existence and homogenization. to appear

[21] D. Giachetti, P. J. Martínez-Aparicio, F. Murat, Homogenization of singular semilinear elliptic equations in domains with small holes, preprint

[22] D. Giachetti, F. Petitta, S. Segura de Leon, A priori estimates for elliptic problems with a strongly singular gradient term and a general datum. Differential and Integral Equations 26 (9/10) (2013), 913-948.

[23] A. C. Lazer and P. J. McKenna, On a singular nonlinear elliptic boundary-value problem. Proc. Amer. Math. Soc. 111 (3), (1991), 721-730.

[24] J. Leray, J. L. Lions, Quelques résultats de Višik sur les problémes elliptiques semilinéaires par les méthodes de Minty et Browder. Bull. Soc. Math. France 93, (1965), 97-107.

[25] M. Montenegro, A. C. Ponce, The sub-supersolution method for weak solutions. Proc. Amer. Math. Soc. 136 (7), (2008), $2429-2438$.

[26] A. C. Ponce, Selected problems on elliptic equations involving measures. arXiv:1204.0668v1.

[27] J. Serrin, Pathological solutions of elliptic differential equations. Ann. Scuola Norm. Sup. Pisa Cl. Sci. 18, (1964), 385-387.

[28] G. Stampacchia, Le problème de Dirichlet pour les équations elliptiques du seconde ordre à coefficientes discontinus. Ann. Inst. Fourier (Grenoble) 15, (1965), 189-258.

[29] Y. Sun, D. Zhang, The role of the power 3 for elliptic equations with negative exponents. Calc. Var. Partial Differential Equations 49 (3-4) (2014), 909-922.

(F. Oliva) Dipartimento di Scienze di Base e Applicate per L' Ingegneria, "Sapienza”, Università di Roma, Via Scarpa 16, 00161 Roma, Italy., FRANCESCO.OLIVA@SBai.uniroma1. IT

(F. Petitta) Dipartimento di Scienze di Base e Applicate per l' Ingegneria, "Sapienza", Università di Roma, Via SCARPa 16, 00161 Roma, ITALY., FRANCESCO.PETITTA@SBAI.UNIROMA1.IT 